days was used to assess total protein intake and protein source. Adequacy of total protein intake $(\mathrm{g} / \mathrm{kg} \mathrm{BW})$ was estimated by comparing with population reference intake of European Food Safety Authority (PRI EFSA).

Of all school-age children involved in this study, 65\% of them had adequate BMI, $22 \%$ were overweight or obese and $13 \%$ were underweight according to sex-standardized WHO BMI-for-age z-scores. Almost all (99.4\%) children exceeded the PRI EFSA recommendation for protein intake. Average daily total protein intake was $2.3 \pm 0.1 \mathrm{~g} / \mathrm{kg}$ BW $(68.4 \pm 1.4$ $\mathrm{g}$ /day), which is about

$249 \pm 72 \%$ of PRI EFSA recommendation. Children's daily animal protein intake $(1.5 \pm 0.04 \mathrm{~g} / \mathrm{kg} \mathrm{BW})$ was twice as much as plant protein $(0.8 \pm 0.02 \mathrm{~g} / \mathrm{kg} \mathrm{BW})$, which was evident from the animal-plant protein ratio $(2.1 \pm 0.1)$. In line with logistic regression, adjusted for energy intake and gender, BMI was negatively associated with total protein intake, as well as with animal protein intake. Accordingly, children who had higher total protein intake $(\beta=-5.087$, OR $0.006,95 \% \mathrm{Cl}$ $0.001-0.04, \mathrm{p}<0.001)$ and animal protein $(\beta=-3.298$, OR $0.037,95 \% \mathrm{Cl} 0.008-0.167, \mathrm{p}<0.001$ ) were less likely to be overweight and obese. No association was observed between $\mathrm{BMI}$ and plant protein intake.

Intrinsically, results suggest that higher total and animal protein intake is associated with lower BMI in school-age children. However, additional studies with different designs would be required to determine the relationship between protein intake (total, animal and plant) and BMI or body composition in school-age children with respect to other lifestyle and environmental factors.

\section{DIAGNOSIS AND MANAGEMENT OF WILSON'S DISEASEIN CHILDREN: A TUNISIAN SINGLE-CENTER EXPERIENCE}

Rania Ben Rabeh*, Latifa Essid, Olfa Bouyahya, Nada Missaoui, Salem Yahyaoui, Sonia Mazigh, Samir Boukthir. Béchir Hamza Children's Hospital

\subsection{6/archdischild-2021-europaediatrics.267}

Goal Wilson's disease (WD) is an autosomal recessive genetic disease characterized by accumulation of copper in the body leading to severe hepatic and neurological damage. It is one of the few genetic diseases that can be treated effectively. The aim of the study was to describe Diagnostic features, therapeutic management and outcome of WD in Tunisian children. Methods Retrospective cohort study of WD cases diagnosed at Tunisian tertiary referral hospital over a period of 10 years (2010 - 2019). Data collection was done from medical records.

Results We collected 20 cases of WD (15 boys and 5 girls). Median age at diagnosis was 8 years [3 -12 years]. Consanguinity was found in 14 patients.

Three patients had a history of WD in the siblings. Clinical presentation was as following: four patients were diagnosed by family screening at a presymptomatic stage, 15 patients presented with hepatic symptoms (jaundice $(n=4)$; hepatomegaly $(n=5)$; clinical signs of portal hypertension $(n=6))$ and one patient presented with hematuria caused by kidney stones (hypercalciuria). Chronic hepatitis, acute hepatitis, liver cirrhosis and fulminant liver failure were observed respectively in two, four, six and four cases. Neurological and psychiatric involvement was noted at diagnosis in $7 / 20$ patients. Kayser Fleischer's ring was observed in three cases.

Proximal tubular involvement was reported in three cases, associated to glomerular involvement in one case. Hematologic disorders were observed in

11/20 cases: pancytopenia $(n=2)$, thrombocytopenia $(n=5)$, Coombs-negative hemolytic anemia $(n=4)$. Thirteen patients had low levels of serum ceruloplasmin, with a median level of $0.12 \mathrm{~g} / \mathrm{L}$ [0.04-0.18]. The 24-hours urinary copper test showed high level in 12 cases with a median level of 3.8 $\mu \mathrm{mol} / 24 \mathrm{~h}$ [1.5-6.72]. D-penicillamine sensitization test was performed in ten cases and was positive in eight cases. Liver biopsy was done in only two cases. Mutation analysis of the ATP7B gene was performed in four cases: no mutation was detected in one case and three patients had homozygous ATB7B mutation (H1069Q). D-penicillamine and pyridoxine were started in all cases.

One patient presented adverse reaction and received zinc acetate (Wilzin).

Most patients were stabilized or improved on chelation therapy, one patient deteriorated and one patient died within the follow up period.

Conclusion Cirrhosis at diagnosis increases the risk of death. Early diagnosis, at a precirrhotic stage was associated with a best prognosis.

\section{ASSESSMENT OF NUTRITIONAL STATUS IN HOSPITALIZED CHILDREN IN TUNISIAN TERTIARY REFERRAL PEDIATRIC HOSPITAL}

Rania Ben Rabeh*, Nada Missaoui, Sonia Mazigh, Sofiène Atiattalah, Salem Yahyaoui, Samir Boukthir. Béchir Hamza Children's Hospital

10.1136/archdischild-2021-europaediatrics.268

Introduction Malnutrition of the hospitalized child is a widespread problem that remains under diagnosed because of the different definitions. The purpose of our work was to assess the nutritional status of the hospitalized children at Tunis Children's Hospital and to determine the factors determining nutritional status.

Methods This is a cross-sectional study in the 'one day' mode conducted three times. We included children aged between 3 months and 13 years and hospitalized for more than 48 hours. We evaluated the auxological parameters according to the curves of the Control Disease Center and the World Health Organization.

Results We enrolled 87 patients. The prevalence of undernutrition was $21 \%$ (18 children). Eight patients (9\%) had chronic undernutrition. Twelve patients were under 24 months of age. Undernutrition was more common among children of rural origin and from poor socio-economic classes. Chronic underlying diseases were present in eleven of undernourished children. Food intake was $57 \%$ in average. Nutritional risk was high in 13 patients.

Acquired undernutrition was observed in 14 patients. Weight loss was more than $5 \%$ in nine of them. Underlying chronic disease, the nutritional risk score and the Waterlow index were significantly correlated with undernutrition.

The prevalence of overweight was 13\% (11 patients). Mean age was 42 months.

Overweight was more common among girls, in rural areas, from poor families with a family history of obesity. The 
prevalence of anemia was 90\%. Only two patients received nutritional support.

Conclusion Malnutrition in the hospital is common in Tunisia but remains under diagnosed and insufficiently supported. This entity deserves further study to determine its impact on health expenditures and to improve its screening and management.

\section{HOW MANY CALORIES ARE ENOUGH?}

Zrinka Šmuljićn ${ }^{*}$ Lana Omerza, Ivan Bambir, Eva Pavić, Ivana Todorić, Ivona Markelić, Andrea Vukić Dugac, Duška Tješić-Drinković. University Hospital Centre Zagreb

10.1136/archdischild-2021-europaediatrics.269

Because of disease complexity, it is challenging to realistically estimate energy needs of cystic fibrosis (CF) patients. During regular dietitian's check-ups, we noticed that CF patients who gained weight or maintained BMI targets consume more calories than are estimated as enough, following the ECFS and ESPEN guidelines. Therefore, we question if $100 \%$ increase of recommended energy intake is enough for some patients.

To support this doubt we calculated patients' energy needs based on Harris-Benedict formula and the level of physical activity, increased them following ESPEN guidelines and compared the recommended to actual intake and thriving.

Best example is a malnourished, picky-eater 12-year girl with poor appetite and impaired lung function (FEV1 29-43\% p.v.). The girl finally agreed to PEG tube in order to increase feeding possibilities when the BMI was $13 \mathrm{~kg} / \mathrm{m} 2$ (-3.03 SD) and FEV1 35\%p.v. At that point, her estimated energy needs were $1900 \mathrm{kcal}$ and according to ESPEN guidelines, the recommended intake was doubled. Because of poor food-intake $(1000-1500 \mathrm{kcal})$, this was achieved mainly through enteral nutritional supplements either orally or via PEG. She still didn't gain weight over a period of 9 months. During her last hospitalisation we reviewed the dietary approach. Her daily energy intake from food didn't surpass $1500 \mathrm{kcal}$ so we increased the enteral feeds to $5000 \mathrm{kcal}$ daily thus mounting energy intake to $\approx 6500 \mathrm{kcal} / 22.5 \mathrm{~kg}$. After the lung function stabilized again (FEV1 37\%p.v.), this high intake was necessary to retain weight gain. Following the same protocol at home, she gained $4 \mathrm{~kg}$ in a month (16.7\% of weight at discharge). Her BMI is now $15.3 \mathrm{~kg} / \mathrm{m} 2(-1.68 \mathrm{SD})$.

Our patient consumes $300-330 \%$ of energy needs of healthy peers. This example emphasizes the importance of the dietitian in the CF team, an individual approach to each patient and 'thinking out of the box'. Maybe it is time to revise the guidelines regarding energy requirements for $\mathrm{CF}$ patients.

\section{ACUTE ESOPHAGEAL NECROSIS IN A 15-YEAR OLD BOY - A CASE REPORT}

Lana Omerza*, Duška Tješić-Drinković, Irena Senečić-Čala, Mirna Natalija Aničić, Dinko Stančić-Rokotov, Jurica Vuković. University Hospital Center Zagreb, Croatia

\subsection{6/archdischild-2021-europaediatrics.270}

Acute esophageal necrosis (black esophagus, Gurvits syndrome) is a rare clinical entity which leads to upper gastrointestinal bleeding. First description dates to 1990, with around115 cases described in the literature. The condition has pathognomonic endoscopic appearance characterized by circumferential black mucosa in the distal esophagus, and discontinuing abruptly at the gastroesophageal junction. The pathogenesis is unclear, apparently multifactorial mucosal ischemia due to low flow vascular state or microvascular thrombosis is predisposing to topical damage by gastric content reflux. It's commonly seen in elderly men, with risk factors like diabetes, malignancy, alcohol consumption, shock, major surgery.

Diagnosis is made endoscopically.

Management requires hemodynamic stabilization, acid suppressive medication with avoidance of nasogastric tube placement. The condition has very poor prognosis, with mortality rate up to $35 \%$, and various complications including strictures and stenosis, perforation with mediastinitis and abscess formation.

Our patient, a 15 year old boy underwent surgery for scoliosis. During the immediate post surgical period he had hematemesis with consequent hemorrhagic shock. He was stabilized (IV fluids, packed red blood cells), nasogastric tube was inserted with evacuation of around $160 \mathrm{~mL}$ of blood and he was referred to our ICU. He required mechanical respiratory support and inotropic medications. Continuous parenteral PPI therapy was commenced.

Black, charcoal-like content was draining from the nasogastric tube, with further deterioration in hemoglobin levels.

Esophagogastroduodenoscopy showed black mucosa of lower esophagus, partly circumferential, partly linear, with cutoff at gastroesophageal junction.

There were no radiological signs of esophageal perforation, bilateral lung consolidates were surrounded by ground-glass interstitial changes.

Patient was kept NPO, on parenteral nutrition, with PPI and antibiotic treatment. He was weaned mechanical ventilation after three days, followed by brief stint of non-invasive respiratory support.

Unfortunately, significant stenosis with stricture formed in the area overlying initial necrosis. After several attempts of endoscopic ballon dilatation, refractory strictures reemerged. Surgical gastrostomy was performed to enable sufficient enteral caloric intake, and bring the patient to ideal physical condition for further treatment.

Planned colonic interposition surgery was not performed because of inadequate length of colon, hence thoracic surgeons performed retrosternal esophagogastroplasty Our patient had no further postoperative complications and was able to establish adequate oral feeding.

Acute esophageal necrosis should be considered as one of the causes of upper gastrointestinal bleeding, especially because its high mortality and complications rate requires immediate and aggressive early management.

\section{RISK FACTORS OF AUTOIMMUNE GASTRITIS IN CHILDREN WITH CELIAC DISEASE}

Natalia Shapovalova*, Valeria Novicova, Ksenia Klikunova, Ekaterina Orlova. Federal State budgetary Institute of Higher Education "Saint-Petersburg State Pediatric Medical University» of the Ministry of Health of the Russian Federation

10.1136/archdischild-2021-europaediatrics.271

The aim was to determine the risk factors of autoimmune gastritis (AG) in children with celiac disease (CD) Materials and 